\title{
ANALYSIS OF THE TREND OF THE DEFORMATION AROUND KANTO REGION ESTIMATED BY TIME SERIES OF PALSAR-2 DATA
}

\author{
T. Nonaka ${ }^{1, *}$, T. Asaka ${ }^{1}$, K. Iwashita ${ }^{1}$, F. Ogushi ${ }^{2}$ \\ ${ }^{1}$ College of Industrial Technology, Nihon University, 1-2-1 Izumi-cho Narashino-shi Chiba, Japan \\ - (nonaka.takashi, asaka.tomohito, iwashita.keishi)@nihon-u.ac.jp \\ ${ }^{2}$ Harris Geospatial Co., 1-20-3 Hongo, Bunkyo, Tokyo, Japan - fogushi@ harris.com
}

Commission III, WG III/3

KEY WORDS: SAR, time-series analysis, SBAS, PALSAR-2, displacement

\begin{abstract}
:
The South Kanto gas field contains natural gas dissolved in water. In the past, large-scale land subsidence has occurred due to the extraction of this natural gas. Therefore, continuous and accurate monitoring for subsidence using satellite remote sensing is essential to prevent any extreme subsidence events, particularly in urban areas, and ensure the safety of residences. In this study, we adopted the small baseline subset (SBAS) method to understand the subsidence trend. We used Advanced Land Observing Satellite (ALOS)-2 Phased Array type L-band Synthetic Aperture Radar (PALSAR-2) data from 2015 to 2019 for this purpose. The results show that the maximum displacement around the Kujyukuri area is more than $10 \mathrm{~mm} /$ year and the mean displacement rate for 2015 to 2019 is $-1.4 \pm 3.2 \mathrm{~mm} /$ year; this value is not as large as some obtained with past PALSAR observations. Comparison of our results with PALSAR observations shows that the number of distributed targets is fewer and the root mean square error of each timeseries displacement value is larger. Further quantitative analysis is required to discuss the reliability of the SBAS-derived displacement rates by PALSAR-2.
\end{abstract}

\section{INTRODUCTION}

In the Kanto region of Japan, a large quantity of natural gas can be found dissolved in brine water, distributed widely across the region. The extraction of this water has caused land subsidence in the past. Since the 1930s, gas production activities have been undertaken by private companies. The large-scale production of gas and iodine began in the mid-1950s in the Kujyukuri area of Kanto region. The production involved the withdrawal of groundwater from depths of 500 to $2400 \mathrm{~m}$. Land subsidence, as a consequence, began to occur in the 1960s and serious land subsidence surveys was initiated at the end of the 1960s. From 1970 to 2005, the maximum accumulated subsidence was 0.85 $\mathrm{m}$ and the subsided area spanned $800 \mathrm{~km}^{2}$ (Shen et al., 2004). Recently, due to the efforts of the administrations and companies, land subsidence has reduced. Land subsidence causes discontinuities in pipelines, the subsidence or tilting of buildings, and intrusion of seawater into freshwater aquifers. Therefore, for sustainability with respect to development, particularly in urban areas, understand land deformation trends as a consequence of human action is vital.

Remote sensing is an effective tool for monitoring large-scale ongoing deformation at low cost, whereas traditional levelling can provide precise measurements. Differential interferometry synthetic aperture radar (DInSAR) is a well-known remote sensing tool for measuring surface deformation. However, spatial and temporal decorrelation severely restrict the use of DInSAR, especially for long-term time-series monitoring of urban areas. The small baseline subset (SBAS) technique (Berardino et al., 2002) is an extension of conventional DInSAR methods. This technique is intended for the analysis of distributed targets. The resultant product resembles one generated by conventional DInSAR; however, a key difference is that SBAS enables the analysis of large time series whereas DInSAR is limited to 2-, 3-, and 4-pass series. The SBAS technique is less sensitive to the number of acquisitions than the persistent scatterers (PS) technique (Ferretti, et al., 2001), because unlike the latter, it exploits spatially distributed coherence instead of exclusively considering single points. However, more acquisition results in better product quality, because it allows better estimation and reduction of the atmospheric component. Further, the SBAS technique is not limited to linear displacement.

While C-band $(5.6 \mathrm{~cm}) \mathrm{SAR}$ data have limitations with respect to vegetation penetration, the longer wavelength L-band (23.6 $\mathrm{cm})$ data can penetrate vegetation and receive backscatter signals from the ground surface. Therefore, in previous studies, the SBAS technique has been developed to monitor deformation around the Kujyukuri area from 2006 to 2010 using 26 Phased Array type L-band Synthetic Aperture Radar (PALSAR) data (Ogushi, 2016). From analysis of the collected data, a displacement of more than $15 \mathrm{~mm} /$ year was observed around the cities of Yachimata and Oamishirasato. Then, they selected 12 validation points and estimated the root mean square errors (RMSEs) of displacement by comparing data with GNSS Earth Observation Network System (GEONET) data. The results showed that the RMSE was about $10 \mathrm{~mm}$. The current PALSAR-2 on the Advanced Land Observing Satellite (ALOS)-2 was launched in 2014 and is expected to be used for many applications, especially disaster management. The improvement of orbit control accuracy made it possible to improve the coherence than with PALSAR. However, knowledge of the difference of displacement accuracy between PALSAR-2 and PALSAR has not been gathered fully in

\footnotetext{
* Corresponding author
} 
previous studies. Therefore, the purpose of this study was to develop a method to monitor deformation using PALSAR-2 data and reveal displacement in the Kujyukuri area by this method. Additionally, we evaluated the accuracy of the time series displacement value by comparing with GEONET data.

\section{STUDY AREA AND USED DATA}

\subsection{Study area}

Figure 1 shows the study area, located inside Chiba Prefecture, part of the Tokyo Metropolitan Area. The Boso Peninsula, in the southern part of Chiba Prefecture, is a large peninsula just east of Tokyo across Tokyo Bay. The total population of the Chiba prefecture was more than 6.2 million in October 2018. The recent urban development around the northwest coastal area of Chiba prefecture has caused overpopulation in that area. The South Kanto gas field, which is spread across the whole of Kanto District around Chiba, extracts natural gas dissolved in water. The amount of natural gas produced there is more than $90 \%$ of the total Japanese production of dissolved-in-water type natural gas. A large amount of natural gas development began in Mobara-shi in the 1950s, and most of the natural gas development in Chiba is still concentrated in the Kujyukuri area even today (Tanno et al., 2006). Figure 2 shows mean subsidence velocity acquired from the levelling data for Chiba Prefecture from 2015 to 2019 (Chiba Prefecture).

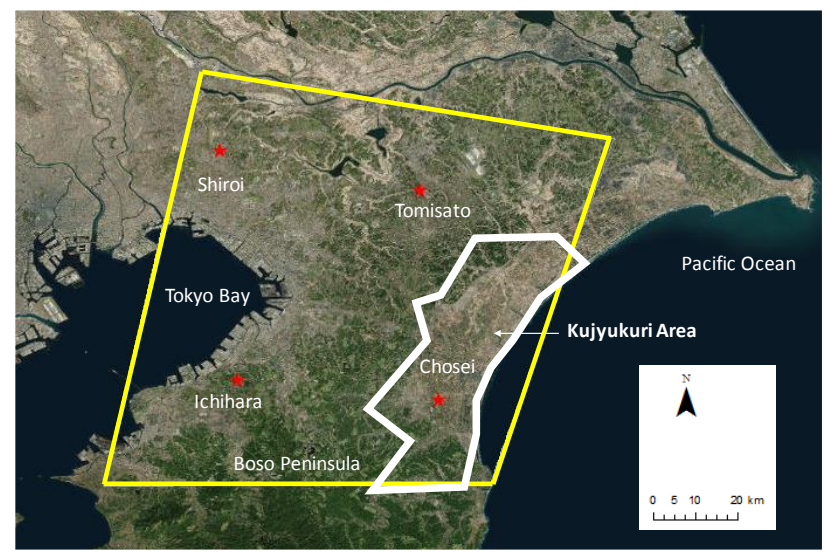

Figure 1. Location of the study area. The red stars indicate GeoNET stations.

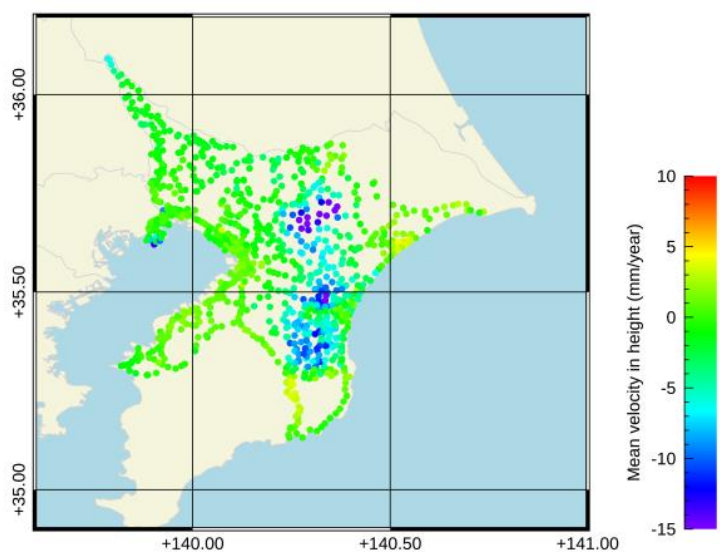

Figure 2. Mean subsidence velocity acquired from the levelling data for Chiba Prefecture from 2015 to 2019

\subsection{Used data}

The PALSAR-2 aboard the ALOS-2 launched in 2014, is currently in operation. It is the latest in a series of Japanese Lband SAR satellites (JERS-1/SAR was launched in 1992, and ALOS/PALSAR was launched in 2006). ALOS-2 was placed in a $628 \mathrm{~km}$ sun-synchronous orbit with local Equator-passing times of noon (descending) and midnight (ascending). The orbit is maintained within a $500 \mathrm{~m}$ accuracy and a frequent 14-day revisit cycle, which makes ALOS-2 three times faster than the previous ALOS (46-day cycle) (Rosenqvist et al., 2014). Because of the limitation in swath width, the scheduled cycle in the basic observation scenario is longer than 14 days. The PALSAR-2 became a unique and highly useful sensor achieving high-resolution and image quality (lowers noise and range ambiguities) by expanding its transmission power and bandwidth. It operates in the $1215-1300 \mathrm{MHz}$ frequency range. Table 1 shows the acquisition parameters used in this study. In this study, we collected 15 PALSAR-2 scenes from January 15, 2015, to March 7, 2019, for the InSAR stacking process. All the scenes were observed in the ultrafine single mode (with $3 \mathrm{~m}$ spatial resolution), the descending direction, and right-looking mode.

The permanent GPS station measurements from the GPS earth observation network system (GEONET)(Geospatial Information Authority of Japan). From the daily GPS data, provided by the GSI of Japan, four points (93018: Shiroi, 93020: Tomisato, 93030: Ichihara, and 93033: Chosei) were selected for validation (Figure 1).

\begin{tabular}{|l|c|}
\hline Band (wavelength) & L-band $(23 \mathrm{~cm})$ \\
Operation mode & Ultrafine \\
Resolution & $3 \mathrm{~m}$ \\
Swathe & $50 \mathrm{~km}$ \\
Off-nadir angle & $32.8^{\circ}$ \\
Orbit direction & Descending \\
Revisit cycle & 14 days \\
Number of images & 15 \\
Temporal coverage & January 2015 to March 2019 \\
\hline
\end{tabular}

Table 1. Acquisition parameters of the ALOS-2 PALSAR-2 dataset

\section{METHOD}

The SARScape module for the ENVI 5.5 software provided by Harris Geospatial Solutions was used to perform the interferometric analysis. The overall SBAS processing workflow included the following steps (Figure 3): creation of connection graph (generating all differential interferograms from the input images stack that fulfil the criteria for temporal and geometric baseline within a given interval in time or for a normal baseline with respect to the critical one), differential interferogram generation (spectral shift and adaptive filtering), phase unwrapping (either with a conventional 2D or with combined 3D approach), orbit refinement and reflattening, first estimation of the average displacement, atmospheric phase screen, and final estimation of the average displacement (SARMap SBAS Tutorial). In this study, a multi-looking factor of three in range and four in the azimuth was used, producing a ground resolution of about $8 \mathrm{~m}$. The topographic phase was removed using a 30-m digital elevation model (DEM) by the Shuttle Radar Topography Mission (SRTM), which has been used for previous PALSAR data processing as well. To smooth the differential phase, the Goldstein filter was applied. The 
minimum cost flow (MCF) network was applied along with the Delaunay method to unwrap the differential interferograms with an unwrapping coherence threshold of 0.30. InSAR estimated displacement is relative to ground control points (GCPs) used during phase displacement conversion. Therefore, the location of GCPs largely determines the quality of InSAR measurements. About a hundred GCPs were selected evenly across an entire image, which was also used for the PALSAR SBAS processing, for refinement and re-flattening. Then, the average displacement rate and residual height correction factors were estimated by inverting a linear system, which includes all measures (one for each interferogram) together with their proportionality coefficients depending on the temporal and geometric baseline of each pair (Pasquali, 2014). A low-pass and high-pass spatial filters were used for the time series images to screen and remove the atmospheric phase component. All of the final displacement measurements were done in the satellite line of sight (LOS) direction and were geocoded in the WGS84 reference ellipsoid.

Figure 4 shows the temporal and spatial baseline distributions of the SAR interferogram from the ALOS-2 PALSAR-2 dataset, in which each acquisition is represented by a diamond associated to an ID number; the green diamonds represent valid acquisitions and the yellow diamonds represent the super master image. For the PALSAR-2 data, we generated 45 interferograms for SBAS processing, with respect to the multi-master images. The threshold criterion for obtaining the absolute maximum normal baselines was half of the critical baseline $(\fallingdotseq 28 \mathrm{~km})$ and that for the absolute maximum temporal baselines was 365 days. The image acquired on March 9, 2017 was chosen as the super master image. The maximum baseline for all pairs was approximately $500 \mathrm{~m}$, which agreed with the specifications.

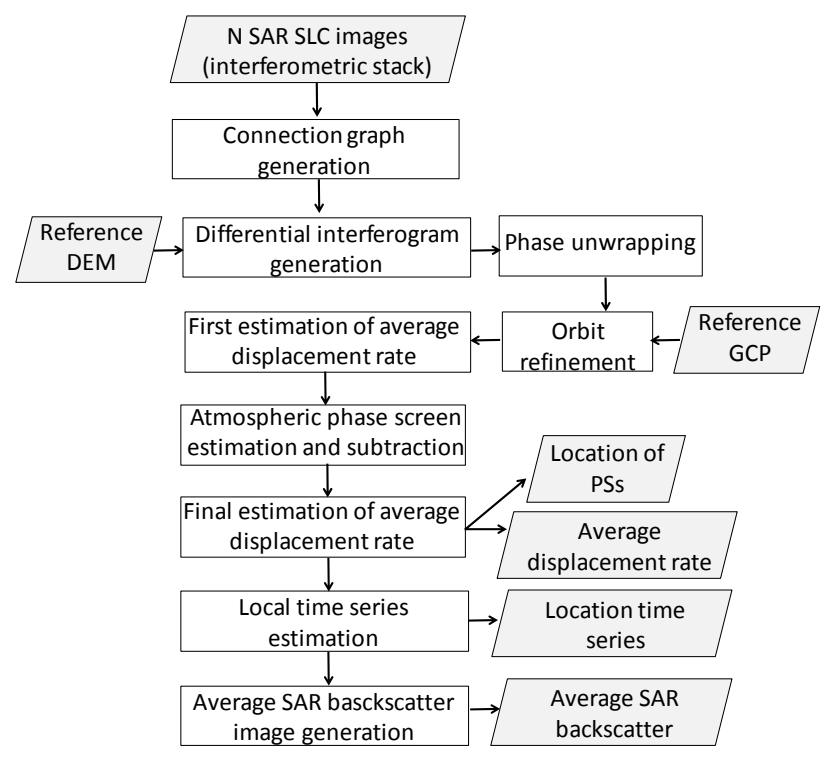

Figure 3. Flowchart of the processing done in this study

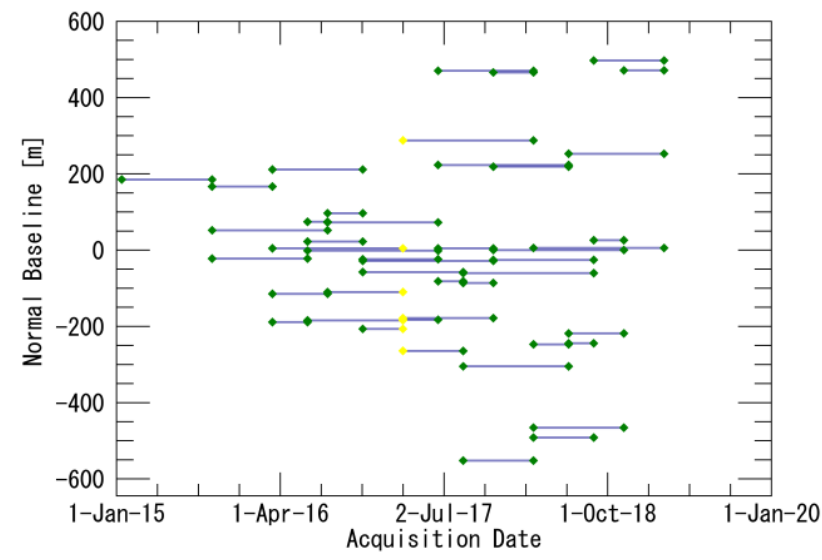

Figure 4. Temporal and spatial baseline distribution of the SAR interferogram acquired from ALOS-2 PALSAR-2 data

\section{RESULTS}

\subsection{Displacement rates}

Almost no displacement was observed around the coast of the Tokyo Bay, while obvious displacement was observed around the Kujyukuri area (Figure 5). The histogram of the estimated displacement velocity for the study area from 2015 to 2019 are shown in Figure 6. The mean displacement rate and standard deviation were -1.4 and $3.2 \mathrm{~mm} / \mathrm{year}$, respectively. The results show that $75 \%$ of the points indicate displacement rates between $-3 \mathrm{~mm} /$ year to $3 \mathrm{~mm} /$ year.

In previous studies, the displacement rates for this area, derived from PALSAR data for 2006 to 2010 , were $-3.3 \pm 5.8 \mathrm{~mm} /$ year. This implies that recent displacement has been less than half that of ten years ago, and the deviation from the mean value is also less, however more than $-10 \mathrm{~mm} /$ year of displacement velocity is seen around cities of Yachimata and Oamishirasato from PALSAR-2. Therefore, the deformation pattern between PALSAR-2 and PALSAR has been consistent, although the acquisition period does not overlap. The acquisition condition for the PALSAR data was almost same as PALSAR-2; a $34.3^{\circ}$ incidence angle and descending path.

Some studies have shown that the displacement values were obtained even for vegetated areas using longer L-band wavelengths, however the distributed targets for PALSAR-2 were not observed by vegetated area due to the lower coherence in these areas. Actually, the number of distributed targets acquired by PALSAR-2 is much less than that acquired by PALSAR. A reason for this could be explained by the difference of interferogram noise. Previous studies have revealed that interferogram noise is proportional to the height of ambiguity, which is defined by Equation (1) (Nonaka et al., 2017). Therefore, one of the reasons for the error is related to the average height of ambiguity, which is higher for the PALSAR-2 data than the PALSAR data.

$$
\frac{d h}{\mathrm{~d} \phi}=\frac{\lambda R \sin \theta}{2 B p}
$$

where $\quad d h / d \phi=$ height of ambiguity

$\lambda=$ wavelength

$R=$ slant-range length

$\theta=$ incidence angle 
$B p=$ perpendicular baseline

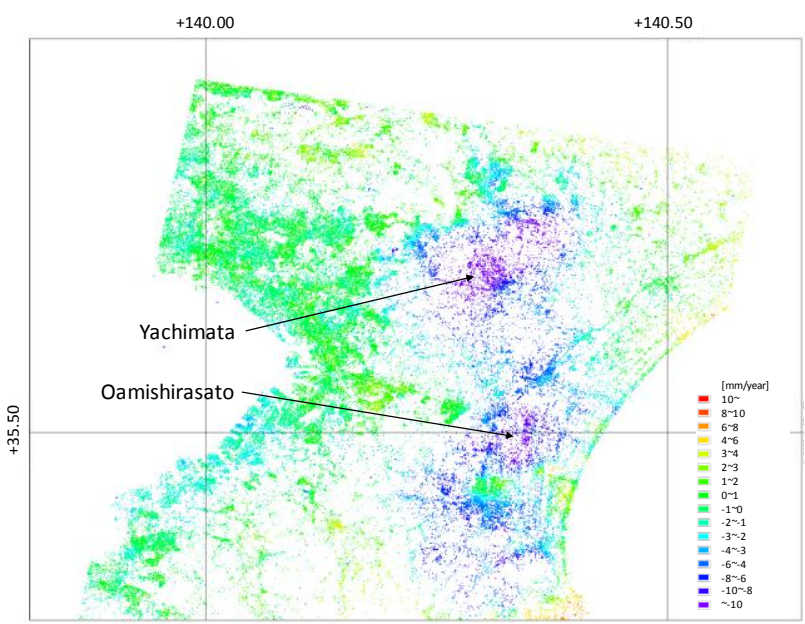

Figure 5. Mean displacement velocity from 2015 to 2019 , derived from the PALSAR-2 data

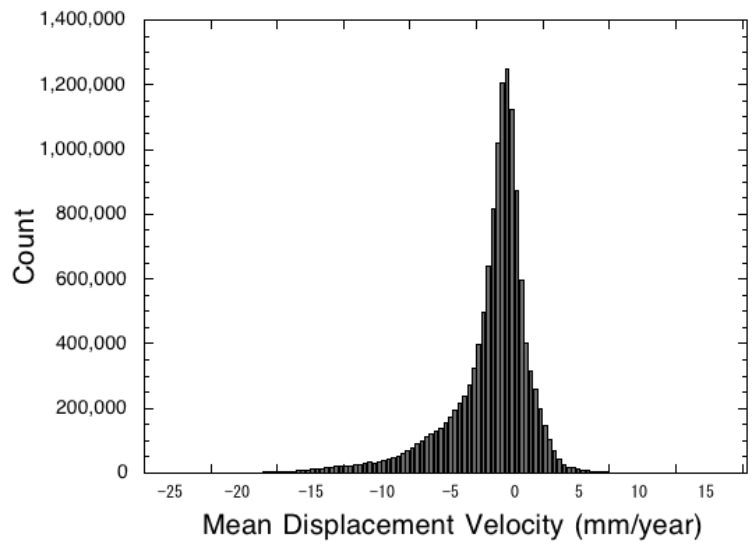

Figure 6. Histogram distribution for the PALSAR-2 derived displacement velocity rates from 2015 to 2019

\subsection{Comparison with GEOMET data}

To assess the accuracy of the InSAR derived results, a quantitative comparison of the time-series displacement values with GEONET data was conducted for four validation points (Figure 1). For the comparison (Figure 7), the GEONET data derived values were converted to those for the line of sight (LOS) direction. The trends of actual displacement in Tomisato and Chosei were continuous and the total amount was about 50 $\mathrm{mm}$ from 2015 to 2019. Several GEONET points inside the study area were not used due to the insignificant value of coherence at those points. Figure 7 shows that the SBAS method underestimated the overall displacement.

The RMSE was $20 \mathrm{~mm}$ for the displacement from 2015 to 2019 , which is twice that of the displacement derived from PALSAR data. The errors in the displacement values were caused by several factors: atmospheric disturbance, interferogram noise, and geometric errors of the sensor (specification: $5 \mathrm{~m}$ ). The errors caused by geometric errors were calculated using a spatial deviation of 3 by 3 pixels around the validation points; it was found to be less than $0.6 \mathrm{~mm}$. (a)

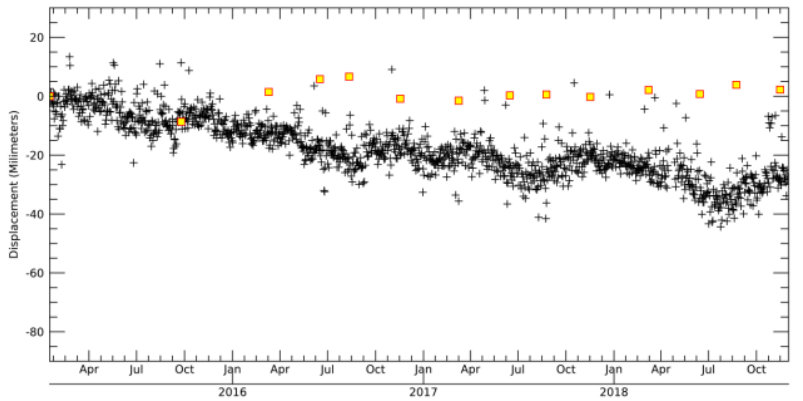

(b)

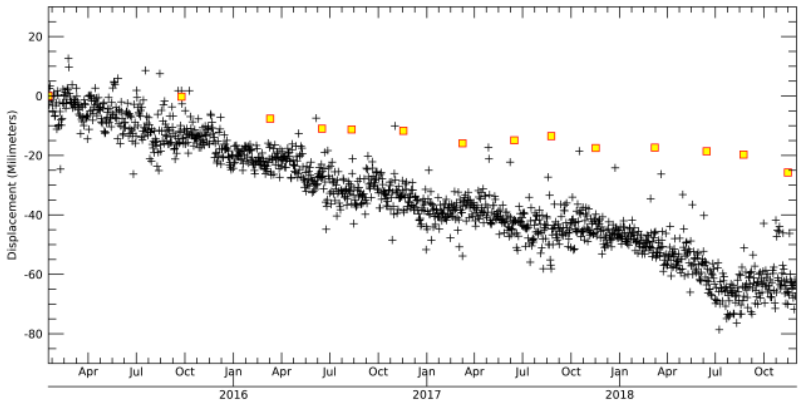

(c)

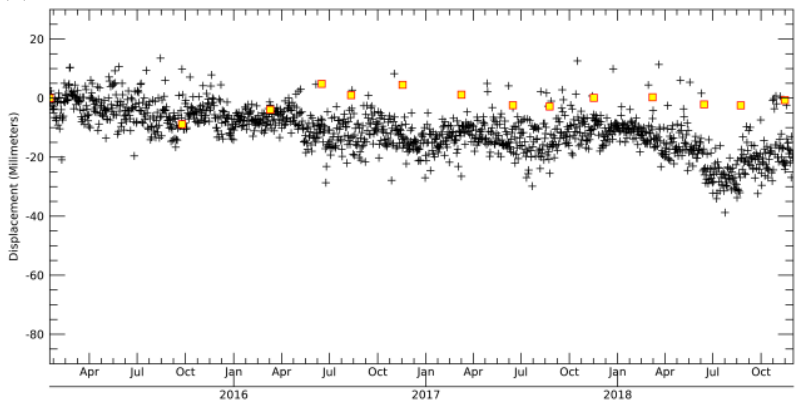

(d)

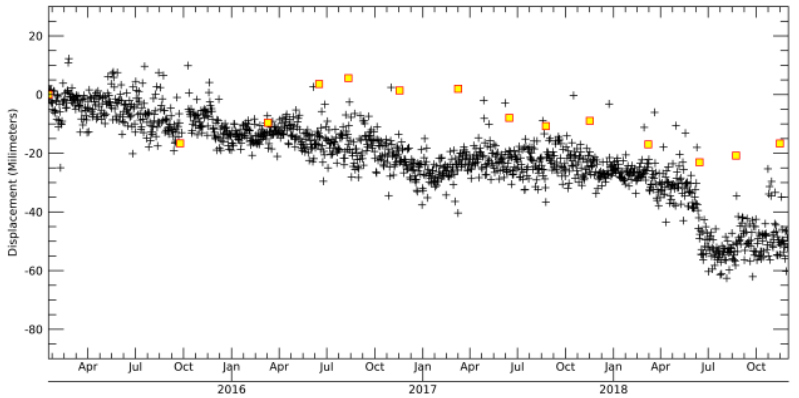

Figure 7. Comparison between the displacement calculated by the SBAS method and that obtained from GEONET data for (a)

Shiroi, (b) Tomisato, (c) Ichihara, and (d) Chosei. The red squares and black pluses show SBAS and GEONET data, respectively 


\section{CONCLUSIONS}

This study estimated the spatial distribution of the land displacement within Chiba Prefecture using time-series satellite SAR data. We adopted the SBAS technique for monitoring the land deformation, and 15 PALSAR-2 data were used. The mean velocity of displacement was found to be approximately 10 mm/year in the Kujyukuri area in the period 2015-2019; however, this value was decreased from that obtained for 10 years previously. The spatial trends of the displacement velocity derived using the SBAS method agreed with subsidence velocity derived from the levelling data. PALSAR-2 underestimated the time-series displacement value compared with GEONET data, and RMSE was about $20 \mathrm{~mm}$. The number of distributed targets acquired by PALSAR-2 is much less than that acquired by PALSAR, though the acquisition conditions, such as threshold coherence, and spatial-temporal coherence, were almost equivalent. One of the reasons was related that PALSAR-2 had larger height of ambiguity. In future study, we quantitatively evaluate the accuracy of the annual rate of displacement determined using PALSAR-2 data by comparison with in-situ levelling survey data.

\section{ACKNOWLEDGEMENTS}

This study was a part of "Temporal analysis of ALOS and ALOS-2 data for monitoring the urban environment" funded by the Japan Aerospace Exploration Agency (JAXA). The authors are grateful to JAXA for providing ALOS-2 PALSAR-2 data. This work was supported by JSPS KAKENHI grant number 17K06468. We would like to thank Editage (www.editage.com) for English language editing.

\section{REFERENCES}

Berardino, P., Fornaro, G., Lanari, R., Sansosti, E., 2002. A new algorithm for surface deformation monitoring based on small baseline differential SAR interferograms, IEEE Trans. Geosci. Remote Sens., 40, 23275-2383.

Chiba Prefecture. Chiba prefecture Levelling Survey Results. Available online:

https://www.pref.chiba.lg.jp/suiho/jibanchinka/torikumi/seikao mote.html (accessed on 25 June 2019).(In Japanese)

Ferretti, A., Prati,C., Rocca, F., 2001. Permanent scatterers in SAR interferometry, IEEE Trans Geosci. Remote Sens., 39, 8 20.

Geospatial Information Authority of Japan, Service to provide GEONET Data, Available online:

http://terras.gsi.go.jp/ (accessed on 25 June 2019).

Nonaka, T., Asaka, T., Iwashita, K., Ogushi, F., 2017. Estimation of phase noise by time series analysis of ALOS-2 PALSAR-2 data, Proc. IGARSS2017 International Geoscience and Remote Sensing Symposium, Fortworth, U.S., July, 2017.

Ogushi, F., 2018. Monitoring of the Kanto plane in Japan with PALSAR-1 and PALSAR-2 with SBAS method, JAXA PI Meeting of Global Environment Observation Mission, Tokyo, Japan, January, 2018.

Pasquali, P., Cantone, A., Riccardi, P., Defilippi, M., Ogushi, F., Gagliano, S., Tamura, M., 2014. Mapping of ground deformations with interferometric stacking techniques, Land
Application of Radar Remote Sensing, Holecz, F., Pasquali, P., Milisavljevic, N., Closson, D., IntechOpen, DOI: $10.5772 / 58225$.

Available online: https://www.intechopen.com/books/landapplications-of-radar-remote-sensing/mapping-of-grounddeformations-with-interferometric-stacking-techniques (accessed on 25 June 2019).

Rosenqvist, A., Shimada, M., Suzuki, S., Ogushi, F., Tadono, T., Watanabe, M., Tsuzuku, K., Watanabe, T., Kamijyo S., Aoki, E., 2014. Operational performance of the ALOS global systematic acquisition strategy and observation plans for ALOS2 PALSAR-2, Remote Sensing of Environment, 155, 3-12.

SARMap SBAS Tutorial. Available online: http://sarmap.ch/tutorials/sbas_tutorial_V_2_0.pdf (accessed on 25 June 2019).

Shen, S., Tohno, I., Nishigaki, M., Miura, N., 2004. Land subsidence due to withdrawal of deep-groundwater, Lowland Technology International, 6(1), 1-8.

Tanno, Y., Maruyama, M., Kannno, R., 2006. Location of gaschemical and metal machinery industries and their business change in Kujukuri area Chiba Prefecture, Annals of human and regional geography, 28, 101-126 (in Japanese). 